\title{
Research and Design of the Web Service Application
}

\author{
Feng Zhou and Xiaoping Feng \\ Jiangxi Technical College of Manufacturing, Department of Information Engineering
}

\section{Keywords: Web Service; Application Integration; Model}

\begin{abstract}
Along with the rapid development of information technology, the integration of enterprise application systems gets more and more attentions, and the main integration technology (RMI, CORBA, and DCOM) relies too heavily on its own protocol due to the inherent model, so the system exists the defect of flexibility and interoperability. Service-oriented architecture is put forward for the enterprise application integration to provide a dynamic, scalable architecture plan. The gradually mature web services SOA provides the technical support for enterprise application integration. Based on the above characteristics, the paper takes use of the standardization of Web services, the characteristics of loosely coupling, high integration to study and analyze the design idea and realization technologies of the application integration model based on Web service, based on which developing an enterprise application integration system, achieving the aim of cross-platform between enterprise and customer, headquarters and branch offices and autonomous, system, and the scalability and interoperability of the application integration system is strengthened obviously.
\end{abstract}

\section{Introduction}

Current society is an era that network economy and network technology develops at high speed and technical innovation emerges constantly. Enterprise and IT organizations are facing increasingly complex business processing and some business process in terms of its life span is short. Different enterprises and IT organizations can choose different application techniques or development environment according to their specific needs. So in such a big environment, the core problem that numerous companies and institutions have encountered is how to integrate and develop applications independently on different computers, how to update the information flexibly and efficiently or construct the distributed software applications that are most suited to the new demand on the basis of the existing software resources. To solve the core problems aforesaid, in recent years, this paper proposes a new architecture, which takes use of standard protocol defining interface, call method, using the components based on internet and the realization of a variety of applications. It finally solves the heterogeneous problem and faces the distributed computing of Web, creating Web service. It effectively solves the problem of interaction among distributed software system s.

Hierarchy integration model based on Web services is proposed in this paper, and the integrated design model, system structure, key technology, development pattern and other key problems are analyzed. This model can meet the characteristics of distributed application integration better with good versatility and compatibility, which can effectively avoid the appearance of Information Island.

\section{Types of Application Integration}

EAI can be divided into different levels of integration according to the different perspective. According to the scope of integration level, enterprise application integration can be divided into the application integration inside an enterprise and application integration between different enterprises. According to the integrated level of technology, from easy to difficult, it can be divided into integration of interface, integration of data, integration of function, and integration of business process:

Integration of interface: it is the simplest in enterprise application integration models. Under the scope of this integration, the integration of a variety of software in general is to use the software 
interface to complete, to form a new, unified display interface, which is the final integration result.

Data integration: the basic idea of data integration model is to integrate the data access of various software components. Data-level integration is often the starting point of application integration. Data-level integration enables different applications to access the shared data, also allow data to move between different data storages. The basic principle of data integration is shown in Fig. 1

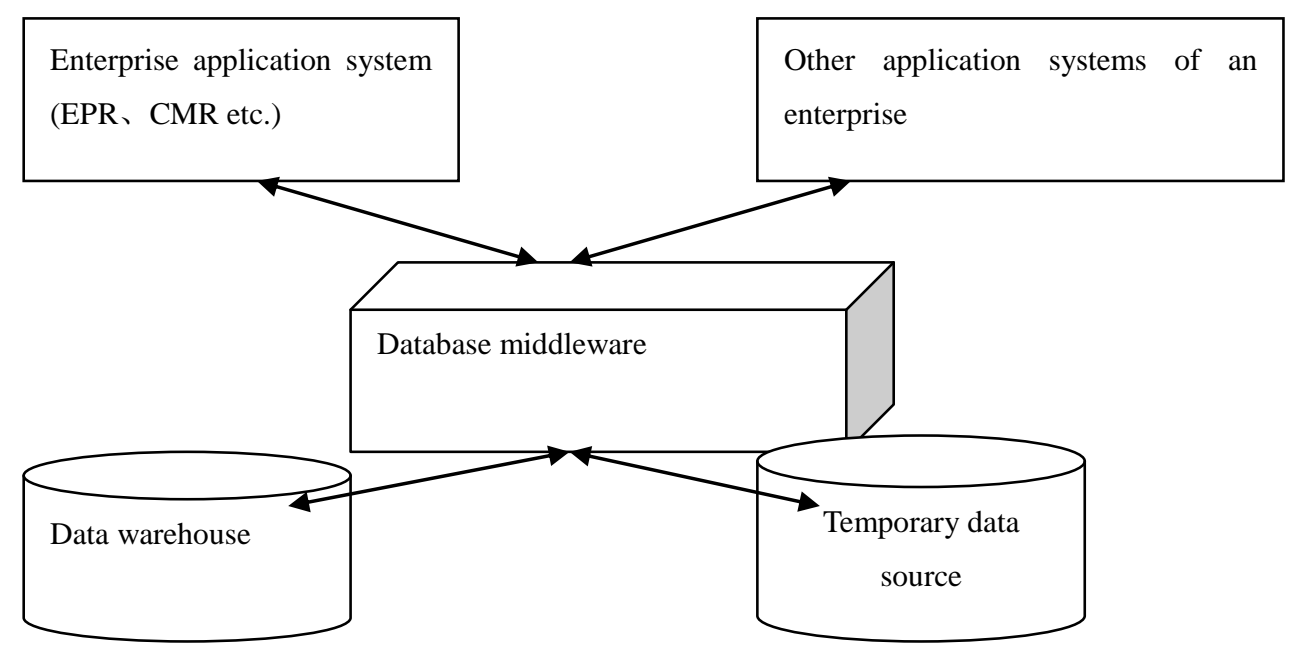

Figure 1. Data integration principle

Integration of function: it is to realize the integration of application on code level, through using the system objects or code logic to realize application system integration. Compared with creating a new logic in the application, it is undoubtedly a more effective method to reuse the existing logic implementation, and access can be customized for each application, including the application of semantic meaning and behavior characteristics, etc.

Integration of business process: it is one of the more advanced process oriented integration, to realize the enterprise business process management through the integration. Create new business process (business process) by integrating the existing IT assets (IT assets (such as data components, applications and services t, etc.).

\section{Basic Concepts of Web Services}

With the development of Web services technology and constant improvement of the standard, application integration technology based on Web service has become popular for the integration research and application of enterprises. Web service technology has become the core implementation technique of enterprise application integration based on SOA at the same time. Now the biggest change for the second generation of Web services is to emphasize more on the reliance of message. The second generation of service inherits the basis agreement of the first generation of agreement, and completely dependent on the SOAP message and adding more extension elements is to extend the SOAP message. Therefore, it is an important problem that how does the second generation of Web services handles the message better. The figure below is Web services stacks based on SOAP message, as shown in Fig. 2: 


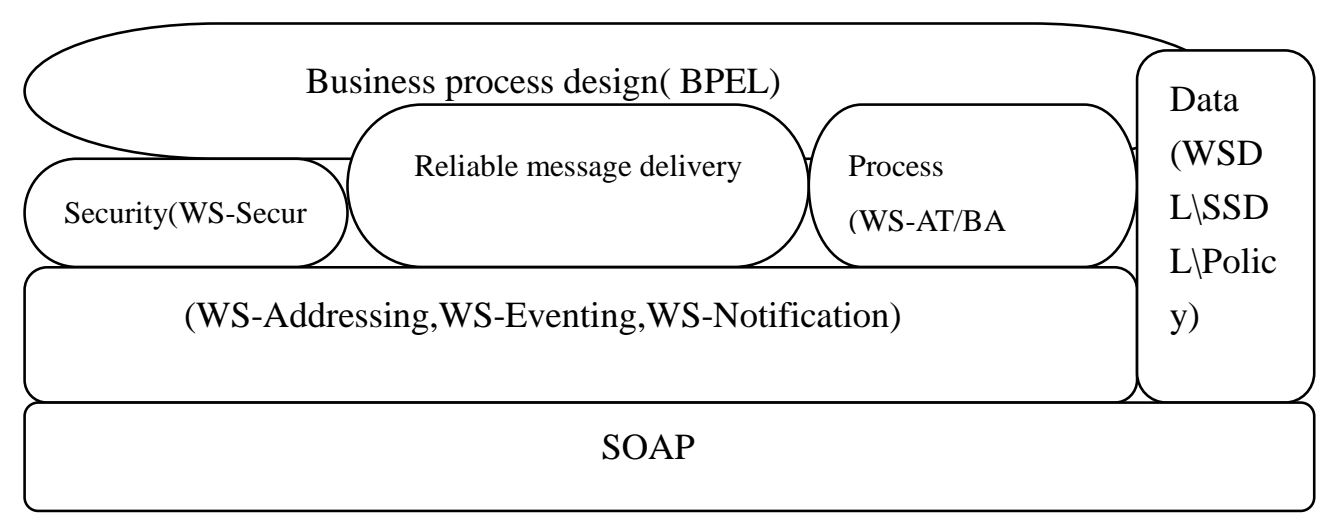

Figure 2. Web services stacks based on SOAP message

Web services have no unified definition until now. In layman's terms, Web service is distributed application components that conform to certain standards and these standards allow them to be accessed outside, and can solve the problem of certain types of industries.

\section{Enterprises Application Integration Design Based on Web Services}

The inner application units of the enterprise are integrated into transparent platform to eliminate the differences inside the unit, among units and data storage methods. The main functions are as follows: A. interaction generality: as the enterprise business constantly changes and frequently between different unit information systems, the interaction of good generality is the assurance that old and new interface conversion does not affect the use of information systems. The whole system network interconnection is finally realized based on the existing information systems of various units to expand. B. Data communication: application integration system can communicate reliably with the internal or external application systems to achieve safe sharing of the subordinate units' information data. C. Generic interface: the information system of each unit inside the enterprise is convenient and quick to connect with the application integration platform, convenient for data exchange between them. The uniform data standard principle is adopted, and unified scheduling interfaces are provided, and each unit can realize the connection and check among enterprises in different places. D. Public management: including isolation communication of private data and public data exchange, generating log, data sharing, query log system, record query, etc. Application integration architecture model as shown in Fig. 3:

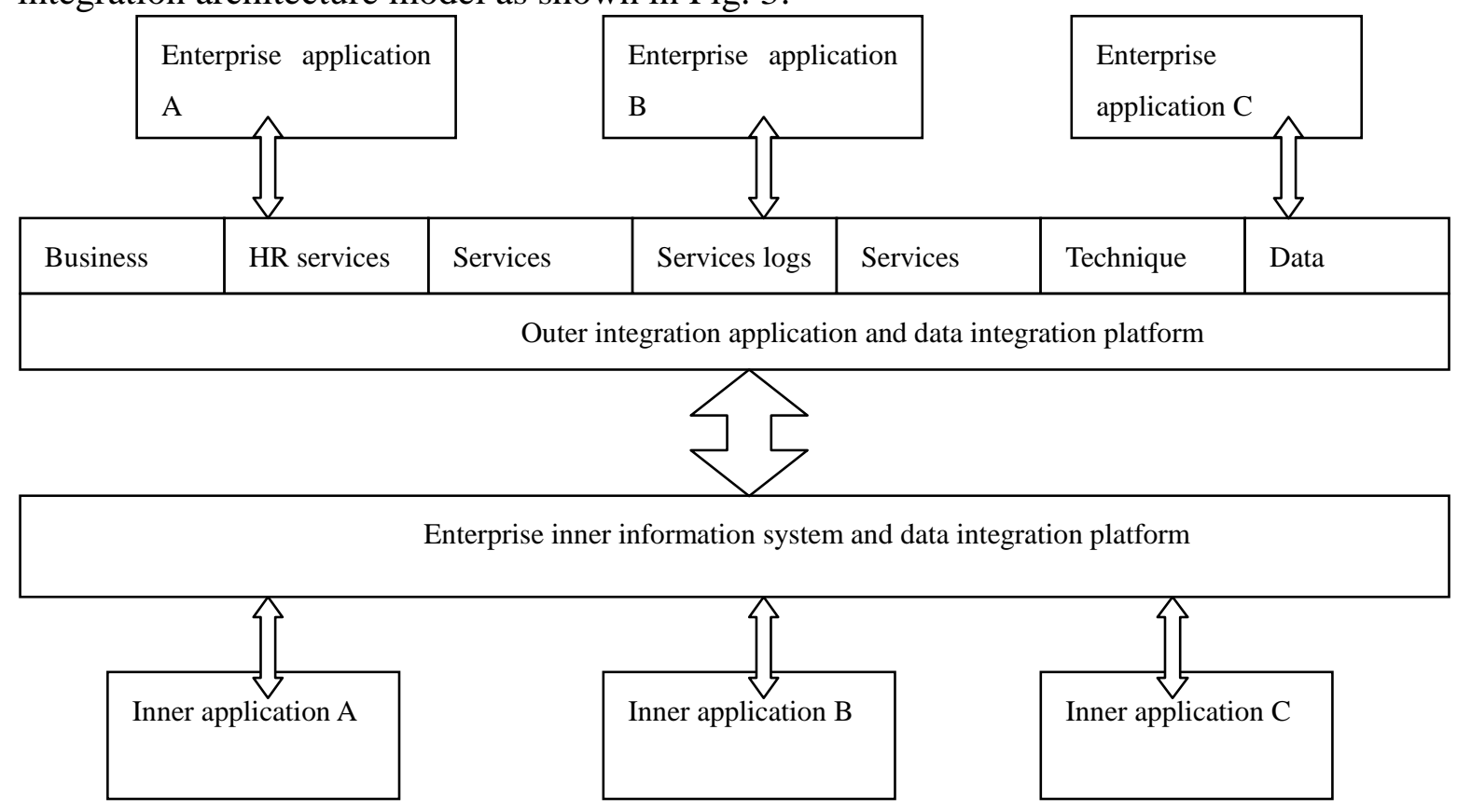


The difference between this system and MIS system of general Web: although built based on B/S system in Web, the general Web MIS system is only a management system, but the concerns can only be management. This paper describes enterprise application integration solutions, which are the comprehensive integration of management, sales, and personnel, using the existing control and code, to implement the comprehensive enterprise application integration. They are the abstract and general integration of the entire enterprise unit.

\section{Conclusion}

Because the traditional application integration system is often confined to the internal integration, usually using point-to-point integration, which brings to the system the shortcoming of poor scalability, on the other hand, when the integration system increases gradually, the relationship among systems will be difficult to manage. At the same time, a large number of redundant information among applications appears, wasting resources and manpower. Based on the analysis of the current development status and shortcomings of enterprise application integration systems, this paper describes the need for a new enterprise application integration system model, in combination with the existing Web service technology, and then puts forward the model of enterprise application integration based on Web service, and analyzes the advantage of the integration model.

\section{References}

[1] Zhang S P. Research and design of remote control system based on Web service[C]// Electronics and Optoelectronics (ICEOE), 2011 International Conference on. IEEE, 2011:V4-428 - V4-429.

[2] Thomas, Horn, Michael, Boutros. E-RNAi: a web application for the multi-species design of RNAi reagents--2010 update. [J]. Nucleic Acids Research, 2010, 38(WebServerissue):W332-9.

[3] Ma J, Hao L, Xiao Y, et al. Research on reconfigurable collaborative design service model and its application $[\mathrm{C}] / /$ Information Management and Engineering, IEEE International Conference on. IEEE, 2010:645 - 649.

[4] Jia S, Guo J, Yang C, et al. Research and Implementation of the Uniform Authentication Based on Web Service. [J]. Applied Informatics \& Communication, 2011, 224:87-92.

[5] Solanki M M R, Shah M S. A Web Service Based Design to Integrate a Web Portal and an ERP System [J]. International Journal of Engineering Research \& Applications, 2014, 4(4).

[6] Tang M X. Research and Implementation of SOA Application Based on Component Technology [J]. Advanced Materials Research, 2014, 926-930:2378-2381.

[7] Zheng Y. Analysis and Design of GRB System Based on WSRF Web Service [J]. Computer \& Information Science, 2012, 5(2).

[8] Manolescu I, Brambilla M, Ceri S, et al. Model-driven design and deployment of service-enabled web applications [J]. Acm Transactions on Internet Technology, 2005, 5(3):439-479.

[9] Koshida T. Design and Implementation of a Primitive Web Service.[C]// International Conference on Information Integration and Web-based Applications \& Services (IIWAS). 2005:73-84.

[10]Xu H X, Lai J M. The Design and Implementation of Baiyangdian Tourism Business System Based on Web Service [J]. Advanced Materials Research, 2010, 143-144:824-827.

[11]Khanbabaei M, Asadi M. Principles of Service-Oriented Architecture and Web Services Application In Order to Implement Service-Oriented Architecture in Software Engineering [J]. Journal of Applied Sciences Research, 2011. 
[12]Liang C. Research and Design of a SOA-based Uniform Application Interface Model [J]. Computer \& Information Technology, 2013. 